Title: Seismic induced damage detection through parallel estimation of force and parameter using improved interacting Particle-Kalman filter

\author{
Authors : Subhamoy Sen \\ Antoine Crinière \\ Laurent Mevel \\ Frederic Cérou \\ Jean Dumoulin
}




\begin{abstract}
Standard filtering techniques for structural parameter estimation assume that the input force either is known exactly or can be replicated using a known white Gaussian model. Unfortunately for structures subjected to seismic excitation, the input time history is unknown and also no previously known representative model is available. This invalidates the aforementioned idealization. To identify seismic induced damage in such structures using filtering techniques, a novel algorithm is proposed to estimate the force as additional state in parallel to the system parameters. Two concurrent filters are employed for parameters and force respectively. For the parameters, interacting ParticleKalman filter [1] is employed targeting systems with correlated noise. Alongside a second filter is employed to estimate the seismic force acting on the structure. The proposal is numerically validated on a sixteen degrees-of-freedom mass-spring-damper system. The estimation results confirm the applicability of the proposed algorithm.
\end{abstract}

\title{
INTRODUCTION
}

Stochastic parameter estimation problems are characteristically categorized as nonlinear stochastic inverse problems. The associated forward problem nonlinearly maps a set of model parameters to the corresponding measurements. Among the existing methods for inversely estimating the parameters, methods defined using a state space formulation are found to be popular [2] due to the relative ease in the estimation. Within this scope, filtering based recursive online system estimation techniques [3] are proved to be more efficient in using the available measurements.

Over the years, Particle Filter (PF) has been established as a good estimator over several other nonlinear variants of Kalman filter (KF) (e.g. Extended or Unscented KF) for nonlinear problems [4]. However, the relatively high computational cost of PF sometimes poses the major concern [5].

In most of the filtering based parameter estimation algorithms, the system states and parameters are usually estimated jointly as an extended state vector [6]. Being model

Subhamoy Sen, Antoine Crinière, Laurent Mevel, Jean Dumoulin - Inria, I4S / IFSTTAR, COSYS, SII, Campus de Beaulieu, 35042 Rennes, France.

Frederic Cérou - Inria, ASPI, Campus de Beaulieu, 35042 Rennes, France. 
based, these filters optimally estimate the parameters of a quasi-steady model of the real dynamic system. Nonetheless, any time variance in the system dynamics may completely diverge the estimation yielding a false or nonphysical solution. By decoupling the estimation of states and parameters (also called as Rao-Blackwellisation [7]) and applying separate but interacting filtering strategies that attempt conditional estimation of states based on parameters and vice versa, time varying systems can be optimally estimated.

Parallel estimation of states and parameters or inputs by coupling two EKF [8] or PF [9] not only ensures better stability in the estimation, but also reduces the computational cost by a great extent. However, while the EKF based algorithms are reported to be not efficient with highly nonlinear systems [10], implementation of PF based dual estimation has concerns over computational expense [5]. It should be noted that, while the parameter estimation is a nonlinear problem, the state estimation focuses on a linear process model. One can thus exploit this by engaging different filter types for state and parameters. Zghal et al. [1] proposed Interacting Particle-Kalman filter (IPKF) which makes a clever use of costly PF for parameter estimation while standard KF handles the linear state estimation problem. The main quality of IPKF is that, with the strategy of decoupling the estimation of states and parameters, the state dimension can always be maintained within reasonable size.

Ultimately, the optimal convergence of any filtering based parameter estimation algorithm depends on the information about the system input. Traditionally, while the time series of the system input is usually unknown, a stationary white Gaussian noise (WGN) model of it is however assumed to be available. Nevertheless, for systems subjected to non-stationary unknown input, traditional approaches fail to provide an optimal solution. Several articles discuss the estimation of system input online [11-13] for mostly time invariant systems. Astroza et al. [14] discussed UKF based joint input-parameter estimation for an unknown yet time invariant system.

Force estimation for a time varying system is however crucial especially for typical structural health monitoring (SHM) problems, since the anomaly in structural system is caused mostly due to some rare events of high magnitude forces for which no prior prediction can be made. Thus in order to develop a robust SHM algorithm, these uncertain forces need to be estimated alongside and communicated to the core SHM algorithm. To achieve this a method is proposed that employs IPKF [1] filter for detecting damage while a second filter parallel to the IPKF estimates the input. Within each KF, the seismic force is considered as WGN and therefore an inverse mapping of the innovation gives a first estimate for the force. Obviously this estimate is not conditioned on the current estimates and therefore is not optimal. This force estimate is then observed through measurements and is subsequently corrected using a second filter i.e., force filter. Updated information about the input is then fed back to the IPKF filter in terms of updating the covariance of the noise process.

\section{PROBLEM DEFINITION}

The governing differential equation for the dynamics of a mechanical system with mass, damping and stiffness matrices being $\mathbf{M}, \mathbf{C}$ and $\mathbf{K}$ respectively subjected to seis- 
mic excitation $\ddot{\mathbf{q}}_{g}(t)$ can be represented as:

$$
\mathbf{M} \ddot{\mathbf{q}}(t)+\mathbf{C} \dot{\mathbf{q}}(t)+\mathbf{K q}(t)=-\mathbf{M} \tau \ddot{\mathbf{q}}_{g}(t)+\mathbf{v}(t)
$$

where $\mathbf{q}(t), \dot{\mathbf{q}}(t)$ and $\ddot{\mathbf{q}}(t)$ are displacement, velocity and acceleration respectively. $\ddot{\mathbf{q}}_{g}(t)$ is the seismic excitation subjected to the structure and $\mathbf{v}(t)$ is the ambient forcing acting on the structure. $\tau$ is a matrix that defines how seismic excitation at some nodes affects the whole structure. The state space representation of the dynamics with the process and measurement noises is:

$$
\begin{aligned}
\dot{\mathbf{x}}(t) & =\mathbf{F}_{c} \mathbf{x}(t)+\mathbf{B}_{c} \mathbf{u}(t)+\mathbf{G}_{c} \mathbf{v}(t) \\
\mathbf{y}(t) & =\mathbf{H}_{c} \mathbf{x}(t)+\mathbf{D}_{c} \mathbf{u}(t)+\mathbf{L}_{c} \mathbf{v}(t)+\mathbf{w}(t)
\end{aligned}
$$

where $\mathbf{u}(t)=\tau \ddot{\mathbf{q}}_{g}(t) . \mathbf{F}_{c}, \mathbf{B}_{c}, \mathbf{H}_{c}$ and $\mathbf{D}_{c}$ are time dependent state, input, measurement and direct transmission matrices respectively defined in continuous time domain. Details of all the matrices are given in the following.

$$
\begin{aligned}
& \mathbf{x}(t)=\{\mathbf{q}(t) \quad \dot{\mathbf{q}}(t)\}^{T} \quad \text { and } \quad \mathbf{F}_{c}=\left[\begin{array}{cc}
0 & \mathbf{I} \\
-\mathbf{M}^{-1} \mathbf{K} & -\mathbf{M}^{-1} \mathbf{C}
\end{array}\right] \\
& \mathbf{B}_{c}=\left[\begin{array}{c}
0 \\
-\mathbf{I}
\end{array}\right], \mathbf{G}_{c}=\left[\begin{array}{c}
0 \\
\mathbf{M}^{-1}
\end{array}\right], \quad \text { and } \quad \mathbf{H}_{c}=\left[\begin{array}{ll}
-\mathbf{M}^{-1} \mathbf{K} & -\mathbf{M}^{-1} \mathbf{C}
\end{array}\right] \text {, } \\
& \mathbf{D}_{c}=-\mathbf{I}, \quad \text { and } \quad \mathbf{L}_{c}=\mathbf{M}^{-1}
\end{aligned}
$$

$\mathbf{v}(t)$ is the process noise and $\mathbf{w}(t)$ is the sensor noise in the measurement.

Since discrete measurements will be used for the estimation, the discrete time formulation of Equation (2) can be presented with $\mathbf{x}_{k}, \mathbf{y}_{k}, \mathbf{F}, \mathbf{B}, \mathbf{G}, \mathbf{H}, \mathbf{D}, \mathbf{L}, \mathbf{u}_{k}, \mathbf{v}_{k}$ and $\mathbf{w}_{k}$ as the discrete time counterparts against their corresponding continuous time entities. The system model is defined as time varying since the stiffness parameters $\theta_{k}$ are varying in time. Subsequently, the corresponding state matrix $\mathbf{F}$ and measurement matrix $\mathbf{H}$ are defined with their dependence on $\theta_{k}$. Mass parameters are however considered to be time invariant and known exactly. Accordingly, the mass matrix and its dependents, i.e., B, G, D and L are assumed to be constant and known from now on.

$$
\begin{aligned}
& \mathbf{x}_{k}=\mathbf{F}\left(\theta_{k}\right) \mathbf{x}_{k-1}+\mathbf{B} \mathbf{u}_{k-1}+\mathbf{G}_{k-1} \\
& \mathbf{y}_{k}=\mathbf{H}\left(\theta_{k}\right) \mathbf{x}_{k}+\left\{\mathbf{D} \mathbf{u}_{k-1}+\mathbf{L v}_{k-1}+\mathbf{w}_{k-1}\right\}
\end{aligned}
$$

The problem assumes that the stochastic properties of ambient forcing $\mathbf{v}_{k}$ and the sensor noise $\mathbf{w}_{k}$ are known and therefore can be modeled as WGN with constant covariance $\mathbf{Q}$ and $\mathbf{R}$ respectively. $\mathbf{u}_{k}$ is the earthquake excitation that has been assumed as Gaussian input. However the covariance of this input is not known a priori and therefore has to be estimated.

\section{PRESENT APPROACH}

Bayesian belief propagation requires an explicit analytical integration to be solved for the entire domain of states. Current problem is nonlinear with respect to parameter 
for which an explicit analytical integration over the entire parameter space is not possible. PF attempts a particle approximation of this integration by propagating the system uncertainty through a cloud of $N$ independent parameter particles $\Xi_{k}=\left[\xi^{1}, \xi^{2}, \cdots, \xi^{N}\right]$ each representing one value for the parameter $\theta_{k}$. The system dynamics is defined by the evolution of this particle set in time as:

$$
\xi_{k}^{i}=\xi_{k-1}^{i}+\mathbb{N}\left(\delta \xi_{k} ; \sigma_{k}^{\xi}\right)
$$

where a Gaussian blurring is performed on $\xi_{k-1}^{i}$ with a shift $\delta \xi_{k}$ and a spread of $\sigma_{k}^{\xi}$.

Eventhough PF is a good estimator for nonlinear systems, the associated high cost is always a major concern. IPKF algorithm, developed by [1] is an efficient approach to handle joint estimation of state and parameter in time varying system. The idea is to nest a bank of linear KFs for state estimation within a PF environment that estimates the parameters. This facilitates employing relatively less expensive linear KF for linear state estimation problem while the costly PF is employed only for parameter estimation. In turn, this approach not only helps lowering the computational cost by reducing the size of the space needs to be explored by particles, but also ensures stability in the estimation of time varying systems by decoupling the estimation of states and parameters.

\section{IPKF algorithm}

In IPKF, the state estimation is performed using a bank of KFs within the PF environment where each of the KFs is associated to one value of the corresponding parameter particles, $\theta_{k}=\xi^{i}$ for which the state estimation is performed. Each of the evolved parameter particles is used to follow the system matrices as $\mathbf{F}_{k}^{i}=\mathbf{F}\left(\theta_{k}=\xi_{k}^{i}\right)$ and $\mathbf{H}_{k}^{i}=\mathbf{H}\left(\theta_{k}=\xi_{k}^{i}\right)$. The stochastic nature of the system input due to ambient and seismic force is defined using a stationary WGN model for $\mathbf{v}_{k}$ with covariance $\mathbf{Q}$ and a non-stationary WGN model of covariance $\mathbf{S}_{k}$ for $\mathbf{u}_{k}$. The cumulative covariance for the process noise is therefore:

$$
\mathbb{Q}_{k}=\mathbf{B S}_{k} \mathbf{B}^{T}+\mathbf{G Q G}
$$

Estimation with IPKF involves propagating the prior $\mathbf{x}_{k-1 \mid k-1}^{i}$ conditioned on current parameter particles to predict $\mathbf{x}_{k \mid k-1}^{i}$ which is then improved using $\mathbf{y}_{\mathbf{k}}$ to obtain posterior

$\mathbf{x}_{k \mid k}^{i}$. This process is repeated for all particles. For each particle, the prediction and correction steps are described in the following.

$$
\begin{array}{cccc}
\text { Prediction: } & \mathbf{x}_{k \mid k-1}^{i}=\mathbf{F}_{k}^{i} \mathbf{x}_{k-1 \mid k-1}^{i} \quad \text { with } & \mathbf{P}_{k \mid k-1}^{i}=\mathbf{F}_{k}^{i} \mathbf{P}_{k-1 \mid k-1}^{i} \mathbf{F}_{k}^{i T}+\mathbb{Q}_{k} \\
\text { Innovation: } & \epsilon_{k}^{i}=\mathbf{y}_{k}-\mathbf{H}_{k}^{i} \mathbf{x}_{k \mid k-1}^{i} & \\
\text { Correction: } & \mathbf{x}_{k \mid k}^{i}=\mathbf{x}_{k \mid k-1}^{i}+\mathbf{K}_{k}^{i} \epsilon_{k}^{i} & \text { with } & \mathbf{P}_{k \mid k}^{i}=\left(\mathbf{I}-\mathbf{K}_{k}^{i} \mathbf{H}_{k}^{i}\right) \mathbf{P}_{k \mid k-1}^{i}
\end{array}
$$

where $\mathbf{K}_{k}^{i}$ is the gain matrix. Each of these KF within IPKF thus yields prediction for the state as $\mathbf{x}_{k \mid k-1}^{i}$ for a parameter particle $\xi_{k}^{i}$.

\section{Force filter}

${ }^{1} A+B \mathbb{N}(\mu ; \sigma)$ means $A+B z$ where $z$ follows $\mathbb{N}(\mu ; \sigma)$ 
Evidently, in order to attain an optimal solution for the parameters with IPKF algorithm, correct stochastic model for $\mathbf{u}_{k}$ is essential. In the present method, the nested KFs consider the seismic force as WGN represented with a non-stationary WGN model with time varying covariance $\mathbf{S}_{k}$ (cf. Equation (5)). Thus to ensure better estimation accuracy, this non-stationary WGN model is required to be estimated online.

Considering the input force $\mathbf{u}_{k}$ to be known, the measurement equation (cf. Equations (3a) and (3b)) for the real system can ideally be described as:

$$
\mathbf{y}_{k}=\mathbf{H}_{k} \mathbf{x}_{k}+\left(\mathbf{H}_{k} \mathbf{B}+\mathbf{D}\right) \mathbf{u}_{k}+\mathbb{N}\left(0,\left\{\left(\mathbf{H}_{k} \mathbf{G}+\mathbf{L}\right) \mathbf{Q}\left(\mathbf{H}_{k} \mathbf{G}+\mathbf{L}\right)^{T}+\mathbf{R}\right\}\right)
$$

However for each nested KF, current estimates (not the true value) of parameter and state affect the uncertainty in the innovation. From Equation (3b), it can be perceived that the innovation uncertainty has dependence on: (i) inexactness in parameter estimates, (ii) measurement uncertainty and (iii) error in force estimates.

The innovation therefore contains the information about the current force. Clearly if an optimal estimation for the force is available, the uncertainty in the innovation can be reduced.

It should be noted here that, if an assumption is made that the innovation $\epsilon_{k}^{i}$ for each particle is solely due to the input, $\mathbf{u}_{k}^{i}$ can be defined as an estimate of seismic force $\mathbf{u}_{k}$ with a Gaussian error model satisfying the following equation:

$$
\epsilon_{k}^{i}=\mathbf{y}_{k}-\mathbf{H}_{k}^{i} \mathbf{x}_{k \mid k-1}^{i}=\left(\mathbf{H}_{k}^{i} \mathbf{B}+\mathbf{D}\right) \mathbf{u}_{k}^{i}+\mathbb{N}\left(0,\left\{\left(\mathbf{H}_{k}^{i} \mathbf{G}+\mathbf{L}\right) \mathbf{Q}\left(\mathbf{H}_{k}^{i} \mathbf{G}+\mathbf{L}\right)^{T}+\mathbf{R}\right\}\right)
$$

Through an inverse mapping, the force can be defined as the combination of the normalized innovation $\epsilon_{k}^{i}$ and a Gaussian error.

$$
\mathbf{u}_{k}^{i}=\left(\mathbf{H}_{k}^{i} \mathbf{B}+\mathbf{D}\right)^{\dagger} \epsilon_{k}^{i}-\left(\mathbf{H}_{k}^{i} \mathbf{B}+\mathbf{D}\right)^{\dagger}\left\{\mathbb{N}\left(0,\left(\mathbf{H}_{k}^{i} \mathbf{G}+\mathbf{L}\right) \mathbf{Q}\left(\mathbf{H}_{k}^{i} \mathbf{G}+\mathbf{L}\right)^{T}+\mathbf{R}\right)\right\}
$$

where $\dagger$ signifies pseudo-inverse. An approximated process model for force estimate at any arbitrary time step $k$ thus can be defined as:

$$
\mathbf{u}_{k}^{i}=\mathbf{u}_{k_{0}}^{i}+\mathbb{N}\left(0, \overline{\mathbf{Q}}_{k}\right)
$$

with $\mathbf{u}_{k_{0}}^{i}=\left(\mathbf{H}_{k}^{i} \mathbf{B}+\mathbf{D}\right)^{\dagger} \epsilon_{k}^{i}$ and $\overline{\mathbf{Q}}_{k}$ being:

$$
\overline{\mathbf{Q}}_{k}=\left(\mathbf{H}_{k}^{i} \mathbf{B}+\mathbf{D}\right)^{\dagger}\left\{\left(\mathbf{H}_{k}^{i} \mathbf{G}+\mathbf{L}\right) \mathbf{Q}\left(\mathbf{H}_{k}^{i} \mathbf{G}+\mathbf{L}\right)^{T}+\mathbf{R}\right\}\left(\mathbf{H}_{k}^{i} \mathbf{B}+\mathbf{D}\right)^{\dagger^{T}}
$$

This prior estimate is conditioned on the propagated system state $\mathbf{x}_{k \mid k-1}^{i}$ and therefore needs to be corrected.

Two different uncertainties are associated to the estimate of $\mathbf{u}_{k \mid k-1}^{i}$ : (i) external of covariance $\overline{\mathbf{Q}}_{k}$ (due to process and measurement noise in the main system), (ii) internal of covariance $\left(\mathbf{P}_{\mathbf{u}}\right)_{k \mid k-1}$ (due to the estimate inaccuracies). The prediction for the mean $\left(\mathbf{u}_{k \mid k-1}^{i}\right)$ and covariance $\left(\left(\mathbf{P}_{\mathbf{u}}\right)_{k \mid k-1}^{i}\right)$ of the force can be obtained as:

$$
\mathbf{u}_{k \mid k-1}^{i}=\mathbf{u}_{k_{0}}^{i} \quad \text { and } \quad\left(\mathbf{P}_{\mathbf{u}}\right)_{k \mid k-1}^{i}=C O V\left[\mathbf{u}_{k}^{i} \mid \mathbf{y}_{1: k-1}\right]=\left(\mathbf{P}_{\mathbf{u}}\right)_{k-1 \mid k-1}^{i}+\overline{\mathbf{Q}}_{k}
$$

Predicted force estimate $\mathbf{u}_{k \mid k-1}^{i}$ is then observed through the measurement to calculate the estimation error as:

$$
e_{k}^{i}=\mathbf{y}_{k}-\mathbf{H}_{k}^{i}\left\{\mathbf{x}_{k \mid k}^{i}+\mathbf{B} \mathbf{u}_{k \mid k-1}^{i}\right\}-\mathbf{D} \mathbf{u}_{k \mid k-1}^{i}+\mathbb{N}(0, \mathbf{R}) ;
$$


with the state estimate mean shifted using the prior estimate of seismic force, i.e., $\mathbf{u}_{k \mid k-1}^{i}$. Finally the force estimates are updated as:

$$
\mathbf{u}_{k \mid k}^{i}=\mathbf{u}_{k \mid k-1}^{i}+\left(\mathbf{K}_{\mathbf{f}}\right)_{k}^{i} e_{k}^{i} \quad \text { and } \quad\left(\mathbf{P}_{\mathbf{u}}\right)_{k \mid k}^{i}=\left[\mathbf{I}-\left(\mathbf{K}_{\mathbf{f}}\right)_{k}^{i}\left(\mathbf{H}_{k}^{i} \mathbf{B}+\mathbf{D}\right)\right]\left(\mathbf{P}_{\mathbf{u}}\right)_{k \mid k-1}^{i}
$$

where $\left(\mathbf{K}_{\mathbf{f}}\right)_{k}^{i}$ is the gain for the $i^{t h}$ force filter at $k^{t h}$ time step which is obtained as:

$$
\left(\mathbf{K}_{\mathbf{f}}\right)_{k}^{i}=\operatorname{COV}\left[\mathbf{u}_{k}^{i} ; \mathbf{y}_{k} \mid \mathbf{y}_{1: k-1}\right] \operatorname{COV}\left[\mathbf{y}_{k} ; \mathbf{y}_{k} \mid \mathbf{y}_{1: k-1}\right]^{-1}
$$

\section{Particle approximation}

In the following, the likelihood of each particle is calculated based on the innovation mean and covariance of each KF. This likelihood information is used to update the normalized weight for each particle. The weight of $i^{t h}$ particle is then estimated as:

$$
w\left(\xi_{k}^{i}\right)=\frac{w\left(\xi_{k-1}^{i}\right) P\left(\mathbf{y}_{k} \mid \theta_{k}=\xi_{k}^{i}\right)}{\sum_{j=1}^{N} w\left(\xi_{k-1}^{j}\right) P\left(\mathbf{y}_{k} \mid \theta_{k}=\xi_{k}^{j}\right)}
$$

The particle approximations for the state, parameter and force are then estimated as:

$$
\mathbf{x}_{k \mid k}=\sum_{i=1}^{N} w\left(\xi_{k}^{i}\right) \mathbf{x}_{k \mid k}^{i} ; \quad \theta_{k}=\sum_{i=1}^{N} w\left(\xi_{k}^{i}\right) \xi_{k}^{i} \quad \text { and } \quad \mathbf{u}_{k \mid k}=\sum_{i=1}^{N} w\left(\xi_{k}^{i}\right) \mathbf{u}_{k \mid k}^{i}
$$

The covariance for $\mathbf{u}_{k}$ is finally updated selecting a window of the past estimated inputs.

\section{NUMERICAL VALIDATION}

Numerical studies are taken up to establish the applicability of this algorithm. The test structure considered here is a 16DOF mass-spring-dashpot system with a seismic excitation. The system is excited with El-centro earthquake excitation (May 18, 1940 in CA, USA, direction North-South) vibration data (Data source: http://peer. berkeley.edu/research/motions/). For the sake of practicality, it has been considered that the earthquake arrived two seconds after the starting of the monitoring and the damage is initiated at the third second of the procedure. All the mass blocks are considered to be same with mass $10 \mathrm{~kg}$ and stiffnesses of all springs are also considered to be $8000 \mathrm{~N} / \mathrm{m}$. The schematic diagram is presented in Figure 1. Damage is incorporated at the sixth DOF by reducing the spring stiffness to $2000 \mathrm{~N} / \mathrm{n}$.

The test structure is subjected to the seismic excitation at the first node. Apart from the seismic forcing, a stationary WGN of variance 1 is applied as ambient excitation acting on all the nodes of the structure. Acceleration response is collected as measurement. Two different case studies are performed considering 8 and 4 measurement channels. The measurements are sampled at a frequency of $50 \mathrm{~Hz}$ for a signal length of 2048 which are subsequently contaminated with stationary WGN of variance 0.1 . The contaminated signal is then put through the proposed approach to simultaneously estimate state, parameter (i.e. indications of damage) and input seismic excitation. 


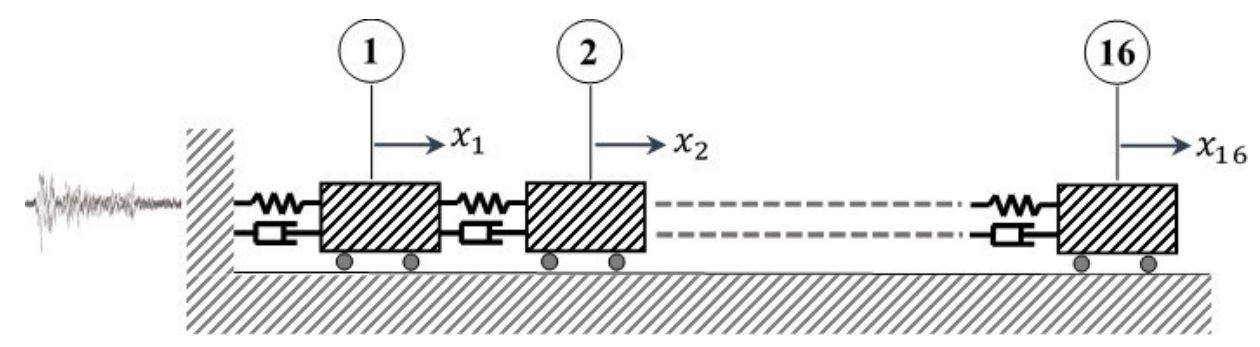

Figure 1. Schematic diagram of the 16 DOF test structure
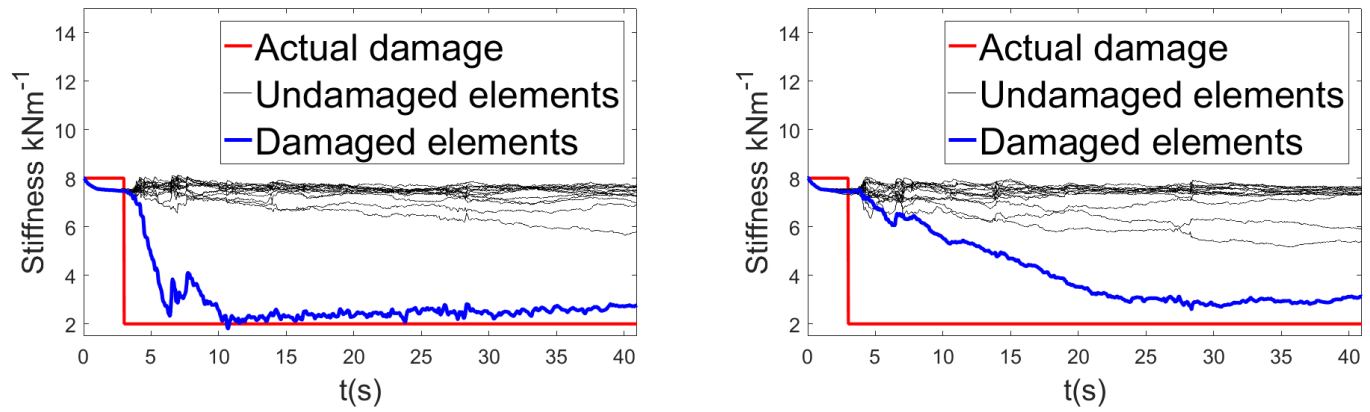

Figure 2. Parameter estimation for 8 (left) and 4 (right) channel measured signal

The IPKF filter is allowed to use only 2000 particles. For the first 100 iterations, a constant value for $\mathbf{S}_{k}$ of $100 N^{2}$ has been supplied.

The results of the parameter estimation presented in Figure 2 and Figure 3 describe the estimation of the forces against their true value. From Figures 2 and 3, it is evident that by reducing the number of the available sensors, the quality of the estimation decreases. However, it can be concluded that for 16 DOF system, a minimum of 4 sensors can still estimate the damage in the system while precisely estimating the input force. It can also be seen, that the damage detection is quite prompt and sufficiently accurate to raise an alarm just as the damage has occurred. Additionally, no case of false alarm during this study has been experienced.
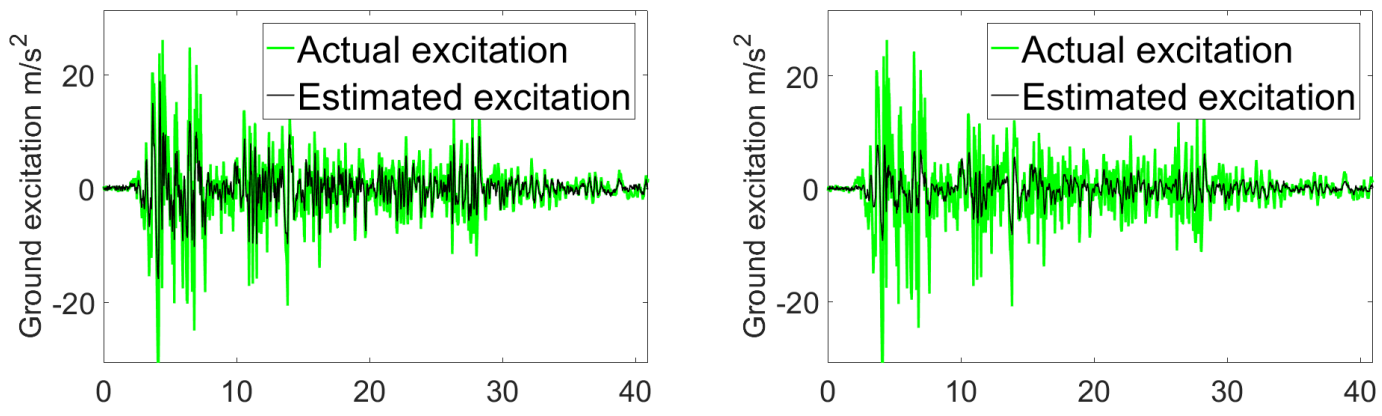

Figure 3. Estimation of input excitation for 8 (left) and 4 (right) channel measured signal 


\section{CONCLUSION}

The proposed article presents a novel particle filter based technique to estimate the parameters of system subjected to an unknown input force of arbitrary distribution using the noise contaminated response measurement. This is done by incorporating a second filter parallel to the state-parameter estimation filter (i.e. IPKF) to identify the force and feed this information back to the previous filter. Numerical experiments are performed on a mass-spring-damper system to demonstrate the proposed method being successful in identifying the damage.

\section{REFERENCES}

1. Zghal, M., L. Mevel, and P. Del Moral. 2014. "Modal parameter estimation using interacting Kalman filter," Mechanical Systems and Signal Processing, 47(1):139-150.

2. Mevel, L., A. Benveniste, M. Basseville, M. Goursat, B. Peeters, H. Van der Auweraer, and A. Vecchio. 2006. "Input/output versus output-only data processing for structural identificationApplication to in-flight data analysis," Journal of Sound and Vibration, 295(3):531-552.

3. Hoshiya, M. and E. Saito. 1984. "Structural identification by extended Kalman filter," Journal of Engineering Mechanics, 110(12):1757-1770.

4. Chatzi, E. N. and A. W. Smyth. 2009. "The unscented Kalman filter and particle filter methods for nonlinear structural system identification with non-collocated heterogeneous sensing," Structural control and health monitoring, 16(1):99-123.

5. Snyder, C., T. Bengtsson, P. Bickel, and J. Anderson. 2008. "Obstacles to high-dimensional particle filtering," Monthly Weather Review, 136(12):4629-4640.

6. Ching, J., J. L. Beck, and K. A. Porter. 2006. "Bayesian state and parameter estimation of uncertain dynamical systems," Probabilistic engineering mechanics, 21(1):81-96.

7. Doucet, A., N. De Freitas, K. Murphy, and S. Russell. 2000. "Rao-Blackwellised particle filtering for dynamic Bayesian networks," in Proceedings of the Sixteenth conference on Uncertainty in artificial intelligence, Morgan Kaufmann Publishers Inc., pp. 176-183.

8. Sen, S. and B. Bhattacharya. 2016. "Online structural damage identification technique using constrained dual extended Kalman filter," Structural Control and Health Monitoring.

9. Azam, S. E., A. Ghisi, and S. Mariani. 2012. "Parallelized sigma-point Kalman filtering for structural dynamics," Computers \& Structures, 92:193-205.

10. Ljung, L. 1979. "Asymptotic behavior of the extended Kalman filter as a parameter estimator for linear systems," IEEE Transactions on Automatic Control, 24(1):36-50.

11. Hsieh, C.-S. 2000. "Robust two-stage Kalman filters for systems with unknown inputs," IEEE Transactions on Automatic Control, 45(12):2374-2378.

12. Lourens, E., C. Papadimitriou, S. Gillijns, E. Reynders, G. De Roeck, and G. Lombaert. 2012. "Joint input-response estimation for structural systems based on reduced-order models and vibration data from a limited number of sensors," Mechanical Systems and Signal Processing, 29:310-327.

13. Azam, S. E., E. Chatzi, and C. Papadimitriou. 2015. "A dual Kalman filter approach for state estimation via output-only acceleration measurements," Mechanical Systems and Signal Processing, 60:866-886.

14. Astroza, R., H. Ebrahimian, Y. Li, and J. P. Conte. 2017. "Bayesian nonlinear structural FE model and seismic input identification for damage assessment of civil structures," Mechanical Systems and Signal Processing, 93:661-687. 
